\title{
Absolute distance interferometry for subaperture stitching of large freeform optics
}

\author{
Jan Spichtinger ${ }^{1, *}$, Michael Schulz ${ }^{1}$, and Gerd Ehret $^{1}$ \\ ${ }^{1}$ Physikalisch-Technische Bundesanstalt, Braunschweig, Germany
}

\begin{abstract}
We are developing a form measurement system for the surfaces of freeform optics and large conventional optics. The specifications of the optics are diameters up to $1.5 \mathrm{~m}$ and radii of curvature down to $10 \mathrm{~m}$. This includes optics like telescope mirror segments and synchrotron optics. Using a Fizeau interferometer, we propose a subaperture stitching method that involves vertically aligning the interferometer's optical axis to the local surface gradient and measuring the absolute distance from the interferometer's reference flat to the specimen. Experimental results for the absolute distance measurement are shown.
\end{abstract}

\section{Introduction}

Measuring the form of an optical surface with a Fizeau interferometer with an aperture smaller than the specimen's diameter makes it necessary to use subaperture stitching [1]. Subaperture stitching is often performed by fitting the offset and the tilt of the subaperture topographies. There are many methods suitable for non-flat optics of different types (e.g. for aspheres, cylinders [2] or freeforms [3]). For arbitrary freeform surfaces with a non-negligible curvature over a large diameter as specified in the abstract, stitching is in general a three-dimensional process. First of all, the interferometer has to be perpendicularly oriented to the local specimen's gradient at each measurement position. By measuring the topography of each subaperture and also the distance from the interferometer's reference flat to the specimen, we will position the topographies from the subaperture measurements in a global coordinate system before stitching. In this way, the stitching process becomes a linearized problem without the need for lateral image registration or computationally intensive multidimensional stitching.

\section{Methods}

\subsection{Setup of the form measurement system}

Our measurement setup [4] will consist of a Fizeau interferometer with a flat reference surface and a specimen, which are mounted on a motion system (see figure 1). The interferometer is attached to a gimbal, which can be moved up and down with a mechanical $\mathrm{z}$-axis. The $\mathrm{z}$-axis is carried by a linear air-bearing $\mathrm{x}$-axis (1500 $\mathrm{mm}$ travel range) on a granite portal. The specimen is placed on a tilt table on top of a rotation table, which is also mounted on an airbearing linear $y$-axis of $1500 \mathrm{~mm}$ in length. Thereby, the

*e-mail: jan.spichtinger@ptb.de
Fizeau interferometer can be perpendicularly oriented to the local specimen surface at any measurement position. The interferometer uses a wavelength shifting laser diode at $633 \mathrm{~nm}$ (referenced on an iodine cell) with a scan range of $250 \mathrm{GHz}$ (referenced on an etalon).

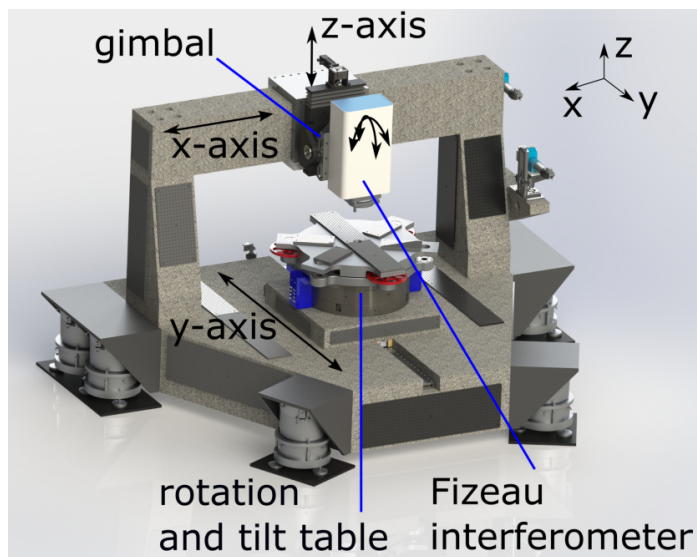

Figure 1. Mechanical setup of the measurement system under development: The arrows represent the possible movements of the Fizeau interferometer and the specimen. The specimen (not shown) is placed on the rotation and tilt table.

\subsection{Topography and absolute distance measurement}

At each measurement position, a wavelength scan is performed with the laser diode of the Fizeau interferometer. During the scan, the intensity changes are measured for each pixel of the interferometer camera (see figure 2). By means of Fourier analysis, the relative topography is obtained by calculating the phase shift of each pixel and unwrapping the resulting spatially resolved phase map. The absolute distance of the interferometer reference flat to the 
specimen, i.e. the cavity length, is calculated from the signal's (temporal) carrier frequency [5]. The resolution of the frequency spectrum obtained by FFT is increased by zero padding. The accuracy of the cavity length measurement is in the order of a few $10 \mu \mathrm{m}$ (see figure 3), while the accuracy of the relative topography measurement is around $10 \mathrm{~nm}$. Only the central pixels of the interferometer camera are used to determine the cavity length.

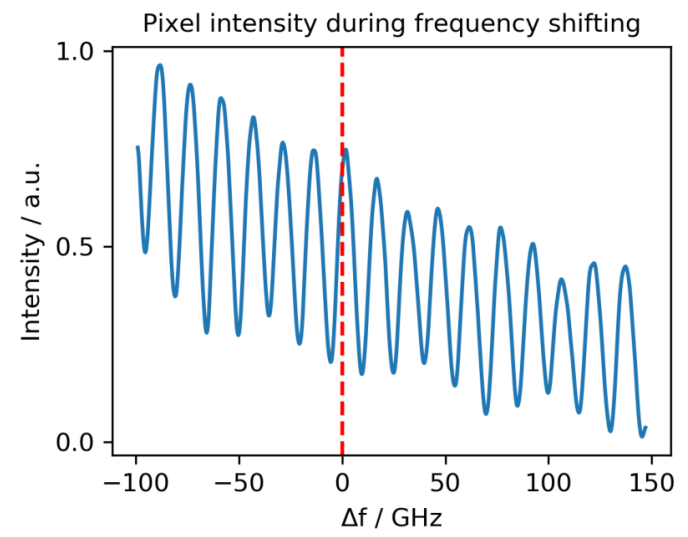

Figure 2. Wavelength shifting interferometry: Measured intensity of a specific pixel in dependence on the frequency shift $\Delta f$ of the laser diode, relative to the centre wavelength of $633 \mathrm{~nm}$. The carrier frequency corresponds to the cavity length.

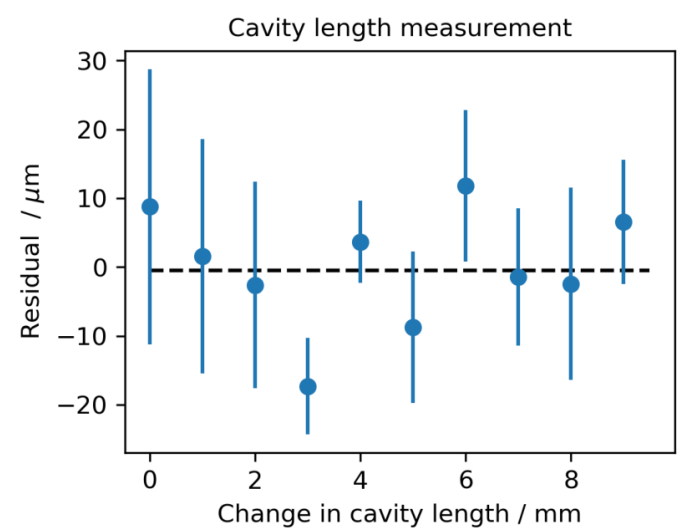

Figure 3. Verifying the absolute distance measurement: A cavity consisting of the Fizeau reference flat and a flat specimen was set up. The cavity length was changed, and the change was measured with a commercial laser interferometer with nanometre precision. The absolute cavity lengths were also measured with the Fizeau interferometer. The figure shows the residuals of the change in cavity length calculated from the absolute distance measurement in comparison to the laser interferometer measurement. Each data point corresponds to the average of 20 measurements with its standard deviation shown as an error bar.

\subsection{Using the absolute distance for stitching}

With the relative topography measurement only, the subtopography could be located anywhere along the optical axis of the interferometer. Having many subaperture measurements with non-parallel optical axes leads to a non-linear problem in the case of global stitching. In the case of sequential stitching, the challenge is to position the first subaperture.

Positioning the topographies in a global coordinate system and fitting only in the z-direction instead of along the optical axis (see figure 4 ) leads to a lateral positioning error $\Delta r$ of the subaperture topography:

$$
\Delta r=\Delta z \cdot \sin (\psi)
$$

with $\Delta z$ : offset of the subaperture determined by the fitting process and $\psi$ : the total angular displacement of the Fizeau interferometer relative to the z-direction of the fit. For the specimens specified at the beginning, the maximal absolute angle is $\psi=4.3^{\circ}$. Therefore, the maximal lateral error will be $\Delta r_{\max }=0.075 \Delta \mathrm{z}$. Since $\Delta \mathrm{z}$ is in the range of a few $10 \mu \mathrm{m}, \Delta \mathrm{r}$ is in the range of a few $\mu \mathrm{m}$.

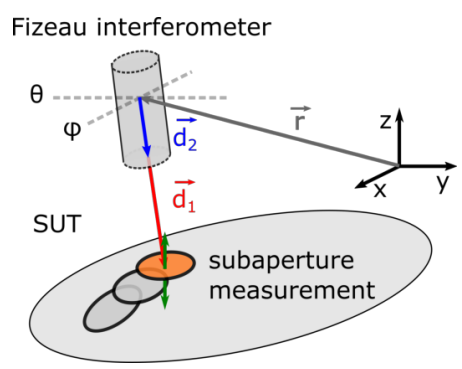

Figure 4. Positioning the topographies from the subaperture measurements: The Fizeau interferometer position $(\vec{r}, \phi, \theta)$ relative to the local surface under test (SUT) is known from the motion system. The subtopographies are positioned with the measured absolute distance $\vec{d}_{1}$ from the reference flat to the SUT and the distance $\vec{d}_{2}$ from the pivot point to the reference flat. The green arrow depicts the z-direction in which the topographies are fitted.

\section{Outlook}

Our next step will be to apply the stitching method with the absolute distance measurement to real measurement data obtained from a measurement system with a smaller movement system than the one presented. The lateral error caused by our approximation of only fitting in the zdirection remains small compared to the typical pixel size of a Fizeau interferometer. This approach is useful as long as the tilt angle range of the interferometer is small enough for the lateral errors to stay within an acceptable limit.

\section{References}

[1] P. Murphy et al., Opt. Photon. News 14, 38-43 (2003)

[2] J. Peng et al., Meas. Sci. Technol. 26, 025204 (2015)

[3] C. Supranowitz et al., Proc. SPIE, 1044818 (2017)

[4] J. Spichtinger et al., Proc. DGaO 2019, ISSN:16148436

[5] A. Cabral, J. Rebordão, Optical Engineering 46(7), 073602 (2007) 